\title{
Active galactic nuclei jets and multiple oblique shock acceleration: starved spectra
}

\author{
A. Meli ${ }^{1}$ and P. L. Biermann ${ }^{2}$ \\ 1 Department of Physics and Astronomy, University of Gent, 4000 Liège, Belgium * \\ e-mail: athina.meli@ugent.be \\ 2 MPI für Radioastronomie, 53121 Bonn, Germany ${ }^{\star \star}$ \\ e-mail: plbiermann@mpifr-bonn.mpg.de
}

Received 9 December 2010 / Accepted 27 April 2013

\begin{abstract}
Context. Shocks in jets and hot spots of active galactic nuclei (AGN) are one prominent class of possible sources of very high-energy cosmic-ray particles (above $10^{18} \mathrm{eV}$ ). Extrapolating their spectrum to their plausible injection energy from some shock implies an enormous hidden energy for a spectrum of index $\sim-2$. Some analyses suggest the particles' injection spectrum at source to be as steep as -2.4 to -2.7 , which exacerbates the problem, by a factor of $10^{6}$. Nevertheless, it seems implausible that more than at the very best $1 / 3$ of the jet energy goes into the required flux of energetic particles, thus one would need to allow for the possibility that there is an energy problem, which we would like to address in this work.

Aims. Sequences of consecutive oblique shock features, or conical shocks, have been theoretically predicted and eventually observed in many AGN jets. Based on that, we use by analogy the Comptonization effect and propose a scenario of a single injection of particles consecutively accelerated by several oblique shocks along the axis of an AGN jet.

Methods. We developed a test-particle approximation Monte Carlo simulations to calculate particle spectra by acceleration at such a shock pattern while monitoring the efficiency of acceleration by calculating differential spectra.

Results. We find that the first shock of a sequence of oblique shocks establishes a low-energy power-law spectrum with $\sim E^{-2.7}$. The following consecutive shocks push the spectrum up in energy, rendering flatter distributions with steep cut-offs, and characteristic depletion at low energies, which could explain the puzzling apparent extra source power.

Conclusions. Our numerical calculations show a variation of spectral indices, a general spectral flattening, and starved spectra, which connect to the relativistic nature of the shocks, the multiple shock acceleration conditions, and the steepness of the magnetic field to the shock normal. This helps in understanding the jet-magnetic field geometry and the irregular or flat spectra observed in many AGN jets (e.g., CenA, 3C 279, PKS 1510-089). Furthermore, the $E^{-2.4}-E^{-2.7}$ ultra-high-energy cosmic-ray injected source spectra claimed by many authors might be explained by the superposition of several, perhaps many, emission sources, all of which end their particle shock-acceleration sequence with flatter, starved spectra produced by two or more consecutive oblique shocks along their jets. It might also imply a mixed component of the accelerated particles above $10^{19} \mathrm{eV}$. Moreover, the present acceleration model can explain the variability of inverted gamma-ray spectra observed in high redshifted flaring extragalactic sources.
\end{abstract}

Key words. acceleration of particles - shock waves

\section{Introduction}

The flux of ultra-high-energy cosmic-rays (UHECRs) at $E \geq$ $10^{9} \mathrm{GeV}$ is believed to arise in plasma shock environments in extragalactic sources; most favorable for this are the hot spots or strong shocks in the jets and radio lobes of active galactic nuclei (AGN) (e.g. Ginzburg \& Syrovatskii 1963; Biermann \& Strittmatter 1987; Rachen \& Biermann 1993) or alternatively, shocks in gamma-ray burst (GRB) environments (e.g. qualitatively by Biermann 1994; quantitatively by Waxman 1995; Vietri 1995). Nevertheless, an absence of neutrinos associated with cosmic-ray acceleration in GRBs (Abbasi et al. 2012) shows that no neutrinos are associated with GRBs.

* IFPA, Department of Astrophysics and Geophysics, University of Liège Belgium and Web Institute of Physics, http://www . wiph.org $\star \star$ Inst. Exp. Nucl. Phys., Karlsruher Institut für Technologie KIT, Germany, Department of Physics \& Astronomy, University of Alabama, Tuscaloosa, AL, USA, Department of Physics, University of Alabama at Huntsville, AL, USA, Department of Physics \& Astronomy, University of Bonn, Germany.
In the mechanism of diffusive shock acceleration, particles repeatedly gain energy in multiple crossings of an astrophysical shock formation through collisions with upstream and downstream magnetic scattering centers, resulting in a powerlaw spectrum extending to the energy of the observed UHECR events. Initially, theoretical developments on particle acceleration were made by Fermi $(1949,1954)$ and Darwin (1954), and during 1970s, the work of Axford et al. (1997), Krymsky (1977), Bell (1978a,b), and Blandford \& Ostriker (1978) established the first-order Fermi mechanism for diffusive particle shock acceleration.

Ultra-high energy measurements by the Auger experiment (Abraham et al. 2007, 2010a,b) have shown that AGN seem a quite plausible candidate sources for UHECRs. Auger also reported a suppression of cosmic-ray flux above $4 \times 10^{19} \mathrm{eV}$. The HiRes experiment reported an observation of the cutoff (Sokolsky et al. 2009). Moreover, Auger measurements indicated a weak statistical trend that supports the idea that very high energy events are localized (Abraham et al. 2010b). In addition, the detection of very hard TeV-energy spectra of high-redshift 
$(z>0.15)$ blazars, such as 3C 279 (Teshima et al. 2009) detected with the MAGIC telescope at a redshift of $z=0.536$, poses one of the most interesting questions in astroparticle physics and shock acceleration efficiency.

It seems implausible that more than at the very best of $1 / 3$ of an AGN jet energy goes into energetic particles (Falcke \& Biermann 1995; Falcke et al. 1995); and so equating the power in UHECRs, adding the additional power hidden at lower energies, and equating it with $1 / 3$ of the observed jet-power, yields a condition on the spectrum: that in turn this would imply a spectrum flatter than $E^{-2.2}$. Thus, one needs to allow for the possibility that there is an energy problem, which we would like, among other aspects, to address in the present study.

The best simple power-law spectral fit to the UHECR $\left(\leq 10^{9} \mathrm{GeV}\right)$ data suggests a spectral index between -2.4 and -2.7 (de Marco \& Stanev 2005; Berezinsky et al. 2006). Thus a total power of $10^{48.5} \times D_{10}^{2} \mathrm{erg} / \mathrm{s}$ is required, where $D_{10} \mathrm{de}$ notes the distance in units of $10 \mathrm{Mpc}$. The prime candidates seem to be CenA and M87 (Ginzburg \& Syrovatskii 1963; Biermann \& Strittmatter 1987). Recently, Gopal-Krishna et al. (2010) obtained an excellent fit for an UHECR signal contribution by CenA, but not just a simple power-law, but a spectrum with a downwards kink. Assuming that up to $1 / 3$ of the total jet power can be supplied, the highest possible power to be provided in UHECR is $10^{42.5} \mathrm{erg} / \mathrm{s}$ for CenA, and $10^{44.5} \mathrm{erg} / \mathrm{s}$ for M87 (e.g. Whysong \& Antonucci 2003; Abdo et al. 2009; Gopal-Krishna et al. 2010), which falls far short of what is required to explain the data. Therefore, one solution would be to consider spectra that maybe are starved at lower energies. We note here that it seems plausible to assume that at around a few $10^{19} \mathrm{eV}$ there are many candidates and only at higher energies there are either very few or perhaps only one source contribution, which would exacerbate the energy problem (i.e. the distance of either CenA or M87 and the interaction with the microwave background is not quite as important, see Greisen 1966; Zatsepin \& Kuzmin 1966).

The observed standing shock features in AGN jets (e.g. Sanders 1983; Gawthorne 2006; Marscher et al. 2008), and the system of repeated shocks observed in the jet structure at vastly discrepant spatial resolutions, for instance in the radio galaxy NGC 6251 (Bridle \& Perley 1984), motivated this work. Moreover, it is well-known from normal quantum statistics that prescribing the number of photons in a box filled with hot gas of temperature $T$, their total energy, and forbidding both creation and destruction of photons, gives highly distorted Planck spectra. These spectra as a function of photon energy $\epsilon$ can be described using a chemical potential, $\mu$ (e.g. Leighton 1959), $N(\epsilon) \sim \mathrm{e}^{-\mu-\epsilon / k T}$. This is called the incomplete Comptonization effect. The process of incomplete Comptonization in the disks of accreting compact objects is one well-known astrophysical application (e.g. Sunyaev 1970; Katz 1976; Rybicki \& Lightman 1993), see Fig. 1. For an insufficient number of available photons, the generated spectrum is depressed at low energies. This entails that a low-energy extension of high-energy spectra may not be realistic, and therefore, we need to allow for depressed low-energy spectra, aiming here to draw an analogy to the possibility that cosmic-ray spectra produced by acceleration in multiple oblique shock structures in AGN jets may also be depressed (starved) in lower energies while extending to higher energies. In the present study, we will show that the first shock from a shock sequence establishes a power-law spectrum with a spectral index of $\sim 2.7$, while the following shocks of the sequence push the particle spectrum up in energy, with flatter distributions manifesting a characteristic flux depletion at lower energies.

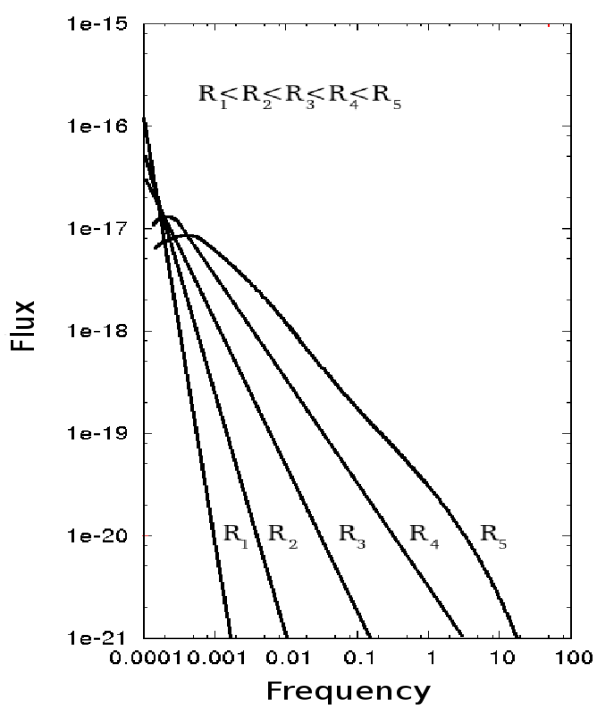

Fig. 1. Radiation from a nonrelativistic Compton photon scattering in a finite gas sphere, where $R_{i}$ denotes different gas radii. Figure reproduced based on Katz (1976).

\section{Jets and multiple oblique shocks}

First attempts to simulate relativistic hydrodynamical jets were made in Marti et al. (1994) and Duncan \& Hughes (1994) for low Mach numbers and in Marti et al. (1995) for high Mach numbers. These studies showed the same trends with the global phenomenology of nonrelativistic jet evolution, but it was shown that relativistic jets propagate more efficiently into the ambient external medium than to a nonrelativistic head jet velocity. Subsequently, Massanglia et al. (1996a) presented more detailed two-dimensional simulations. Three-dimensional simulations (e.g. Arnold \& Arnett 1986; Clarke 1996) also revealed internal oblique shock structures in light or moderately supersonic jets. Among many insightful findings regarding the structure of a relativistic jet, it was found that when a high pressure cocoon develops, it squeezes the jet and drives shock waves into it, which reflect on the axis and form a conical (in other words, an oblique shock sequence) shape. The aspect of this interaction depends on the Mach number. Furthermore, one could establish a relationship between a best angle $(\alpha)$ of the cone between its tangential shock surface and the flow parallel to the jet axis expressed in Lorentz factor $\Gamma$ as $\sin \alpha \propto 1 / \Gamma$. The critical parameter was found to be the inclination of the conical shocks that determined the thrust behind the jet head, meaning in planar jet geometry: oblique shocks must have a low inclination angle to the axis to produce a strong acceleration effect.

The physics of magnetohydrodynamic (MHD) shocks topology in magnetized flows is still poorly understood in the plasma and astrophysical community. Efforts for understanding the jet physics via MHD simulations in one-dimensional (1D), 2D and $3 \mathrm{D}$ are currently under investigation. Although jet dynamics is inherently 3D, much of the physics of these jets can be obtained from simple 1D simulations of flow along a cylindrical shell. For example, Cheung et al. (2007) demonstrated that observed proper motions of forward (superluminal) and reverse (subluminal) knots can be precisely reproduced by a $1 \mathrm{D}$ relativistic MHD simulation model. A very interesting work was presented in a series of papers by Jones et al. (2001), Tregillis et al. (2001a,b, 2004), O'Neil et al. (2006), and others where the authors have conducted extensive studies of high-resolution 2D and 3D MHD simulations of supersonic jets, exploring the influence of the jet Mach number and the ambient medium 
on jet propagation and energy deposition over long distances. Obviously, only 3D simulations can reproduce the obliquity of the features in some jets observed with very high angular resolution, e.g. NGC 6251, 3C 66B, M 87.

Throughout this work, we assume a sequence of oblique shocks along the jet axis (as depicted in Fig. 4), following the reconfinement mechanism (Sanders 1983). Sanders showed by the method of characteristics that this process is a typical property of transversely observed jets. The internal pressure in a free jet decreases rapidly with distance from the AGN nucleus, and therefore the jet would be expected to come into pressure equilibrium with the ambient medium. The reconfinement shocks allow the jet flow to adjust to the outside pressure which remains supersonic. These shocks are almost periodically repeated, contacting the jet circumference, and they are mostly subluminal as long as the flow remains supersonic.

The reconfinement mechanism and the solutions of a hydrodynamic wind (e.g. Fukue \& Okada 1990) show that a jet flow moves through multiple critical points, passing from subsonic to supersonic and vice versa several times, with formation of shock structures. This mechanism is an attempt to recover the strength of a weak jet flow, which would mean that one would have repeated formations of superluminal to subluminal shocks, until the critical point where the outside pressure totally overpowers the pressure of the jet in the deceleration phase.

Observations of polarized synchrotron radiation in AGN jets (e.g. Agudo et al. 2001) indicate stationary conical components in the observer frame in parsec-scale jets, which could be identified as recollimation shocks. We note that systems of repeated oblique (conical) shocks are observed in jet structures at vastly discrepant spatial resolutions, for instance in the radio galaxy NGC 6251 (Bridle \& Perley 1984), interpreting this as a quasiself-similar pattern, with the local scales all proportional to radial distance along the jet. Marscher et al. (2008, 2010) showed high-resolution radio images and optical polarization measurements of the BL Lac-type object PKS 1510-089, which revealed a bright feature in its jet that caused a double flare of radiation from optical frequencies to $\mathrm{TeV}$ gamma-ray energies as well as a delayed outburst at radio wavelengths. It was concluded that the observational event started in a region with a helical magnetic field that was identified with the acceleration and collimation zone predicted in theoretical works, and the variability in the observed brightness was due to emission by the plasma excited by a conical standing shock wave. Furthermore, observations of the jet structure of M87 (Walker et al. 2008) and PKS 1510-089 (Marscher et al. 2008) near the central black hole indicated that while there can be moving shocks between 10 and 1000 Schwarzschild-radii $\left(r_{\mathrm{s}}\right)$, the first strong stationary shock occurs at $\sim 3000 r_{\mathrm{s}}$, as previously proposed by Markoff et al. (2005) and confirmed by Britzen et al. (2008) for the case of the BL Lac-type object S5 1803+784.

The evidence of helical jets transverse rotation measure gradients across AGN jets was predicted by Blandford (1993), and among others found by Gabuzda et al. (2004) in BL Lacs. Brown et al. (2009) found similar evidence in an FRI radio galaxy 3C 78. Asada et al. (2008) performed multi-frequency polarimetry for the quasar NRAO 140 using the VLBA. The observations revealed helical magnetic components associated with the jet itself. Evidence for a co-existence of shocks and helical magnetic fields was reported in Keppens et al. (2008). It is important to note that using MHD simulations these authors explored the morphology of AGN jets by studying propagation characteristics of a series of highly relativistic, helically magnetized jets. Among other results they showed that the magnetic helicity changes at internal cross-shocks, which then act to repeatedly re-accelerate the jet. Moreover, Nakamura et al. (2010) showed that extragalactic jets are the result of MHD shocks produced in helically twisted, magnetized relativistic outflows.

Additionally, we note that state-of-the art laboratory experiments of supersonic highly conductive plasma flows (e.g. Lebedev 2005; Ampleford et al. 2008) have also shown the development of conical shocks forming at the base and along the axis of the developed jet.

The topology we assume here considers the axisymmetric reconfinement of shocks as a sequence of shocks with inclination angles $a, b, c$ and $d$ within a supersonic AGN jet with a helical magnetic field, as shown in Fig. 4. Specifically, we assume that particle acceleration can take place in such internal shocks as theoretically predicted or observed in e.g. PKS 1510-089 and NGC 6251.

The paper is structured as follows: In Sect. 3 we present our numerical method and discuss the results of single and multipleshock acceleration studies. In Sect. 4 we conclude by discussing our findings.

\section{Simulations and results}

\subsection{Numerical method}

We have extended the relativistic particle shock acceleration code of Meli \& Quenby (2003b) to multiple oblique shocks, see the simulation framework shown in Fig. 5. In principle, a Monte Carlo code gives a solution to the time-independent Boltzmann equation by

$\Gamma(V+v \mu) \frac{\partial f}{\partial x}=\left.\frac{\partial f}{\partial t}\right|_{c}$,

where a steady-state is assumed in the shock rest frame, $V$ is the fluid velocity, $v$ the velocity of the particle, $\Gamma$ is the Lorentz factor of the fluid frame, $\mu=\cos \theta$ the cosine of the particle's pitch angle $\theta$, and $\partial f /\left.\partial t\right|_{c}$ is the collision operator.

The use of a Monte Carlo technique to solve Eq. (1) is dependent on the assumption that the collisions represent scattering in pitch angle and that the scattering is elastic in the fluid frame where there is no residual electric field. Because it is assumed that the Alfvén waves have a lower speed than the plasma flow itself, the scattering is elastic in the fluid frame. A phase-averaged distribution function is appropriate to the diffusion approximation we employed, which uses many small angle scatterings. The first-order Fermi (diffusive) acceleration is simulated provided there is a shock front, where the particles' guiding-center undergoes consecutive scatterings with the assumed magnetized media. In each shock-crossing particles gain an amount of energy prescribed by the appropriate jump-condition equations.

A justification for the test-particle approach is the work of Bell (1978a,b) who has shown that "thin" subshocks appear even in the nonlinear regime, so at some energy above the plasma $\Gamma$ value, the accelerated particles may be dynamically unimportant while they re-cross the discontinuity. Another way of arriving at the test-particle regime is to inject particles well above the plasma particle energy when they are dynamically unimportant and thus require the seed particles to have already been preaccelerated. Particles are injected upstream toward the shock and are allowed to scatter in the respective fluid rest frames with their basic motion described by a guiding center approximation.

In a shocked environment, flow into and out of the shock discontinuity is not along the shock normal (Begelman \& Kirk 1990), but a transformation is possible into the so-called normal shock frame (NSH) to render the flow along the normal. Furthermore, an important Lorentz transformation from the 
NSH frame to the so-called de Hoffmann-Teller frame (HT) (de Hoffmann \& Teller 1950) can apply. In the HT frame the electric field $\boldsymbol{E}=0$. Thus, one can study the diffusive shock acceleration mechanism in an electric-field-free reference frame, boosting from the NSH frame by a speed $\beta_{\mathrm{HT}}$ along the shock surface as, $\beta_{\mathrm{HT}} \leq \beta_{\mathrm{NSH}} \tan \psi$. By inspecting the last equation, it becomes obvious that given relativistic shocks, $\beta_{\mathrm{NSH}} \sim 1$ for all angles smaller than $\tan \psi=1$, otherwise the velocity $\beta_{\mathrm{HT}}$ will be greater than one. This physical causality gives rise to a classification of relativistic shocks into two categories: i) the so called subluminal shock, whose inclination is $\tan \psi \leq 1$ (for these low-inclination relativistic shocks the first-order Fermi (diffusive) acceleration applies in the electric-field-free HT frame). ii) The superluminal shock when its inclination is $\tan \psi>1$ (in superluminal shocks particles are accelerated by the so-called, shock-drift acceleration mechanism in the presence of the electric field, see Armstrong \& Decker 1979).

Standard theory poses the conservation of the first adiabatic invariant in the HT frame to determine reflection or transmission of the particles. Reflection of particles during diffusive acceleration is important since it contributes to the overall efficiency of acceleration by frame transformations. In the HT frame the allowed and forbidden angles for transmission depend only on the input pitch and phase, not on rigidity, hence the results of Hudson (1965) apply in our model. In the relativistic shock situation anisotropy renders the input to the shock from upstream very anisotropic in pitch angle, but as was shown in Hudson (1965) and Meli (2002), is an acceptable approximation to randomize phase before transforming to the HT frame and then to use the adiabatic invariant to decide on reflection/transmission. While in the subluminal case, particle transmission at the shock can be decided in the HT frame by employing conservation of the first adiabatic invariant. In the superluminal case computations are followed entirely in the fluid rest frames with reference to the NSH frame simply employed to check whether upstream or downstream shock conditions apply (Meli \& Quenby 2003b). On the other hand, for the superluminal shock conditions, where the physical picture of the shock-drift acceleration mechanism applies, it is necessary to abandon the guiding center approximation when the trajectories begin to intersect the shock surface. For this case, we considered a helical trajectory motion of each test-particle of momentum $p$, in the fluid frame, upstream or downstream, where the velocity coordinates $\left(u_{x}, u_{y}, u_{z}\right)$ of the particle are calculated in 3D space (e.g. Meli \& Quenby 2003b). Begelman \& Kirk (1990) pointed out that in the blast wave frame the turbulence can be isotropic and many shock stationary frame configurations can be superluminal. Nevertheless, many polarization observations show that chaotic magnetic fields prevail at distances larger than a few parsecs, but they should be statistically anisotropic to produce a net linear polarization, as discussed in Korchakov \& Syrovatskii (1962). To this end, Laing (1980) has pointed out that a chaotic magnetic field that initially is isotropic becomes anisotropic after crossing a shock front because of occurred plasma compression. Moreover, Meli \& Quenby (2003b) showed that a transformation from an initially isotropic rest frame distribution to an accelerated flow frame leads to a co-moving relativistic plasma frame field distribution lying close to the flow vector. This condition allows for a range of subluminal situations when viewed in the shock frame.

The basic coordinate system to describe a shock is a Cartesian system $x y z$, where the shock plane lies on the $y z$ plane. In principle, a shock is placed at $x=0$, while $x<0$ corresponds to the upstream region and $x>0$ to the downstream one. The direction of the flow in the shock rest frame is in the

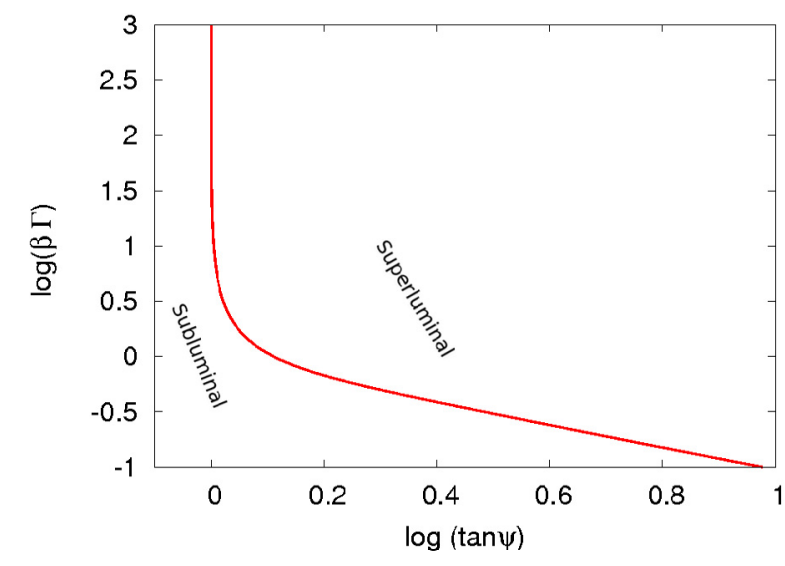

Fig. 2. Allowed shock inclination angles of a Lorentz transformation into the de Hoffmann-Teller frame following $\beta_{\mathrm{HT}} \leq \beta_{\mathrm{NSH}} \tan \psi$. The ordinate shows the logarithm of the $\beta \Gamma$ product of the intersection point velocity of the flow to the shock surface, while the abscissa shows the $\log (\tan \psi)$ where $\psi$ is the angle between the flow and the shock normal in the normal shock frame. In the de Hoffmann-Teller frame the vector of the velocity of the flow and the vector of the magnetic field are parallel to each other.

positive direction, that is from upstream to downstream. The reference frames used during the simulations are the upstream and downstream fluid rest frames, the NSH frame, and the HT frame. Viewing in the NSH frame (see Fig. 3) and assuming that the flow velocity vector is parallel to the shock normal one sees that $i+a=90^{\circ}$ and $\psi+i=90^{\circ}$ thus $\psi=a$, which means that the inclination of the shock surface to the flow consequently indicates the inclination of the shock to the jet axis. Therefore, as long as $\psi$ is less or equal to $45^{\circ}$, the HT frame transformation is possible (Figs. 2, 3). The scattering operator in our simulations is treated via a pitch angle scattering approach, see the appendix and Meli et al. (2008).

We allowed particles with pitch angle $\delta \theta$ chosen at random within $1 / \Gamma \leq \delta \theta \leq 10 / \Gamma$ (where $\Gamma$ is the shock's Lorentz factor). Furthermore, we allowed $\lambda=10 r_{\mathrm{g}}$ ( $r_{\mathrm{g}}$ the particle's gyroradius), which implies a mild turbulence, which is justifiable since there are mostly low magnetized tenuous plasmas in AGN jets farther away than 3000 Schwarzschild-radii. For more details on turbulence media and particle scattering kinematics used in this work, see the appendix.

In the present model we assumed four consecutive oblique shock planes, e.g., with half-opening angles $a, b, c$ and $d$ with respect to the axis $x$. The magnetic field strength was set to $B=10^{-4}$ Gauss, typical for AGN jets. In Fig. 5 one sees the overall jet topology and shock geometries (not to scale) as the framework of our simulation. In the present model we assumed that the overall area of the four shocks occupies a space of $100 \mathrm{pc}$, because most AGN jets have maximum lengths of a few hundreds pc. We mention here that a particle's gyroradius of, e.g. an energy $\sim 10^{11} \mathrm{GeV}$, within a typical jet magnetic field strength of $B \sim 10^{-4}$ Gauss will be $\leq 100 \mathrm{pc}$. The particles differential spectrum is recorded downstream at a distance $d=25 \mathrm{pc}$ from each shock, as shown in Fig. 5. The energy spectra are calculated in the shock frame downstream at $d=25 \mathrm{pc}$ from each shock and normalized to $\mathrm{GeV}$ units, assuming protons as the primary accelerated population. Moreover, particles leave the system if they reach a maximum momentum boundary set at $E_{\max }=10^{11.5} \mathrm{GeV}$.

We started the simulation off the NSH frame as previously described. A Lorentz transformation allocates the magnetic field vector perpendicular to the jet axis, i.e., with an inclination to 

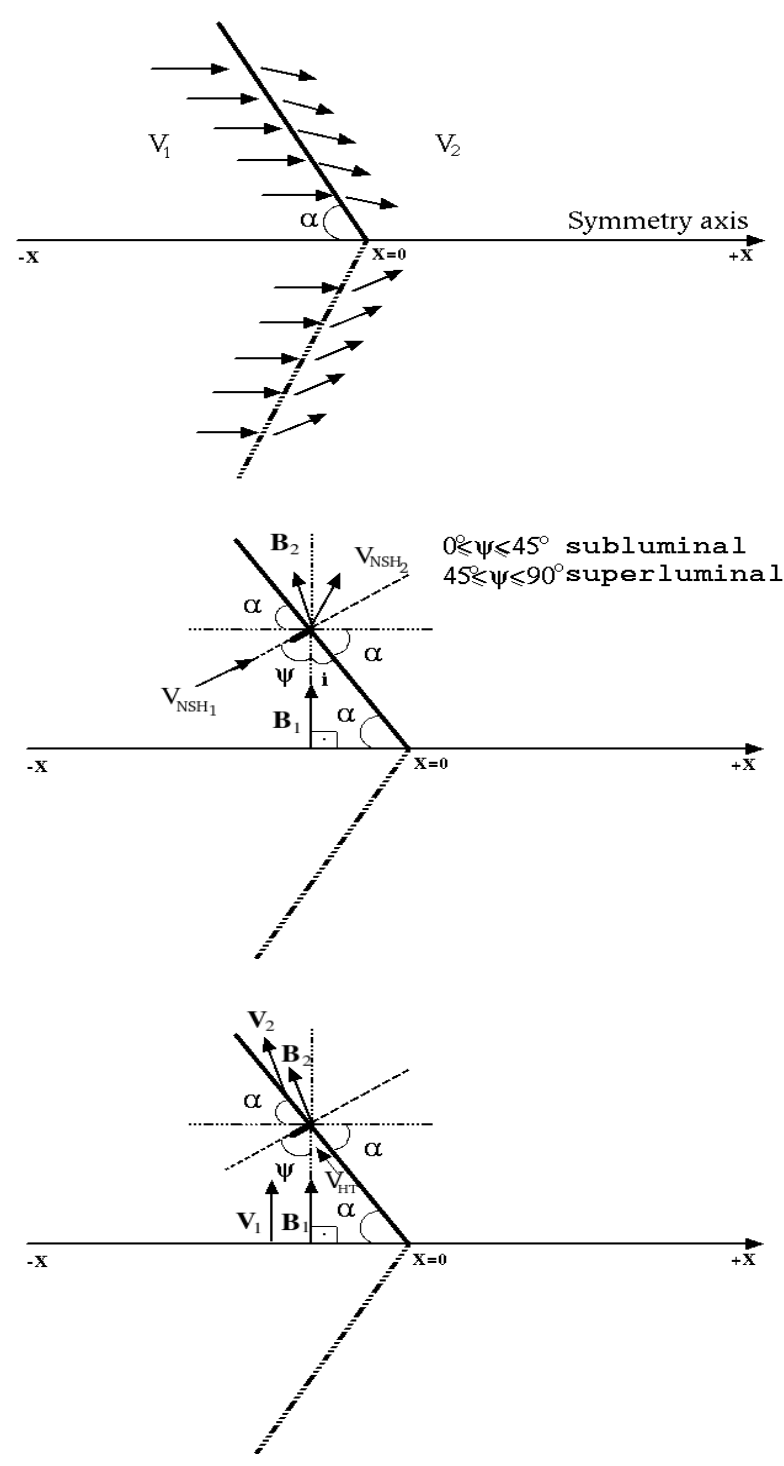

Fig. 3. Left panel: general topology of the flow in a conical shock. Right panel: a conical shock as viewed in the normal-shock-frame (NSH), where the vector of the upstream flow velocity is parallel to the shock normal. Viewing closely the topology in NSH frame, one sees that $90^{\circ}=$ $i+a$ and $90^{\circ}=\psi+i$ then $\psi=a$. The magnetic field is oblique to the shock surface by definition, so here, in order to facilitate our view clearer on the geometry of a conical shock in a local Cartesian system, we assume the case of the magnetic field vector $(\boldsymbol{B})$, perpendicular to the jet axis thus inclined to the shock normal. Bottom panel: the same conical shock as it viewed after the Lorentz transformation into the de Hoffmann-Teller frame (HT). In this frame the velocity vector is placed parallel to the vector of the magnetic field as was viewed in the NSH frame above. By this transformation there is no electric field $\mathrm{E}$ in this frame.

the shock normal, see Fig. 3. We assumed that the first shock of the sequence occurs at about 3000 Schwarzschild-radii from the AGN black hole (Markoff et al. 2005; Becker \& Biermann 2009). Injection of a fixed number $N_{i}$ of particles took place upstream towards the first shock. The key point here is that in the following three shocks of the sequence, acceleration occurs for the same number of particles without an injection of new particles, in analogy to the incomplete Comptonization effect (Leighton 1959), see Sect. 2.

Particles were assigned a random phase so that a 3D transformation of momentum vectors could be achieved between the

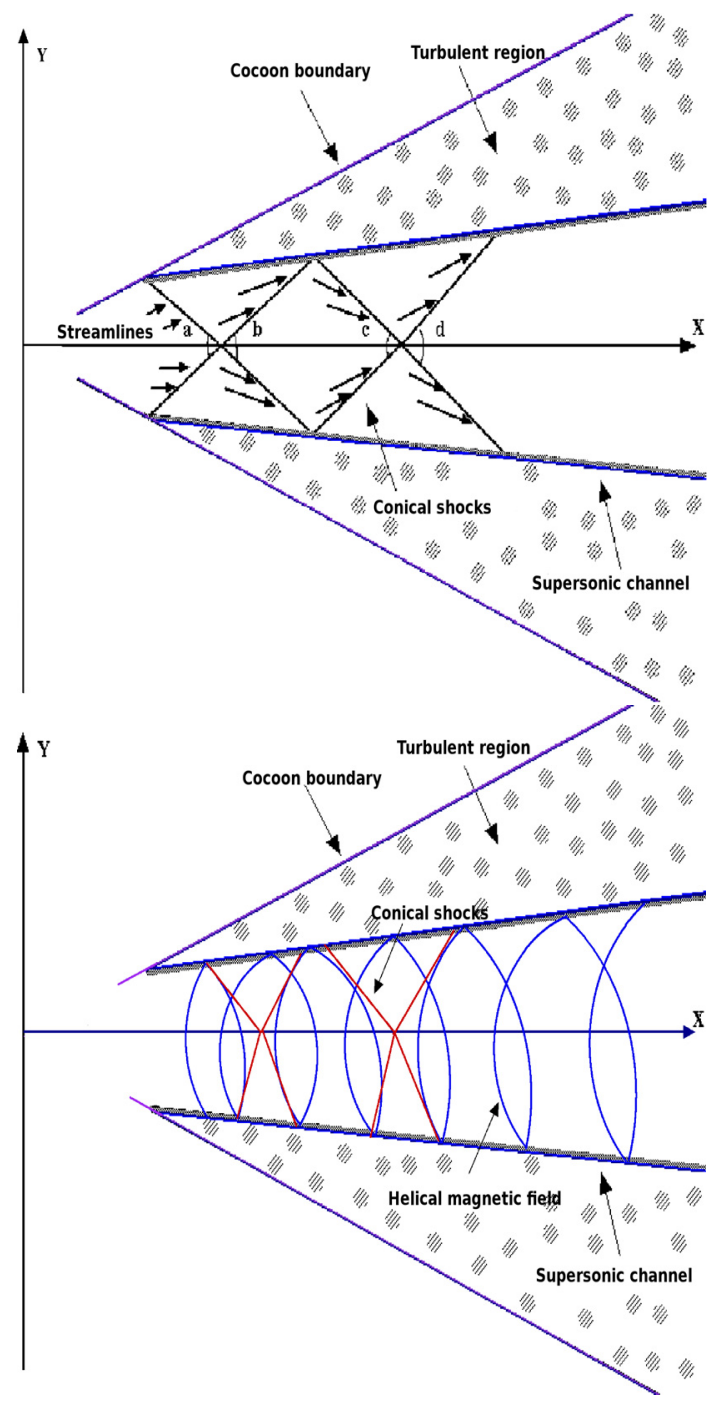

Fig. 4. Concept of conical shock structures and helical magnetic field topologies: Left: repeated conical shock structure of opening angles $a, b, c$ and $d$, in an AGN jet viewed at the shock frame. Right: an overall view of the topology of the magnetic field in an AGN jet (not to scale).

fluid and HT frames. Away from the shock, the guiding center approximation used so that a test-particle moving a distance $d$ along a field line at $\psi$ to the shock normal has a probability of collision within $d$ given by $P(d)=1-\exp (-d / \lambda)=R$, in the plasma frame, where the random number $R$ is $0 \leq R \leq 1$. Weighting the probability by the current in the field direction $\mu=\cos \theta$ yields $h=-\lambda \mu \ln R$. The pitch angle is measured in the local fluid frame, while a value $x_{i}$ gives the distance of the particles to the shock front, where the shock is assumed to be placed at $x=0$, as we mentioned. Furthermore, $x_{i}$ is defined in the shock rest frame and the model assumes variability in only one spatial dimension.

Particle splitting was used to improve statistics, meaning that when a particle reached a certain energy, it was replaced by two daughter-particles, which are otherwise identical to the primary particle, but have only half of the original statistical weight. We assumed that there is no significant escape of particles perpendicular the jet axis off the reconfinement boundary, see Fig. 5, because the particles remain inside the supersonic channel and propagated to the right along the $x$-axis. This is justifiable by the mechanism of shear jet boundary acceleration 


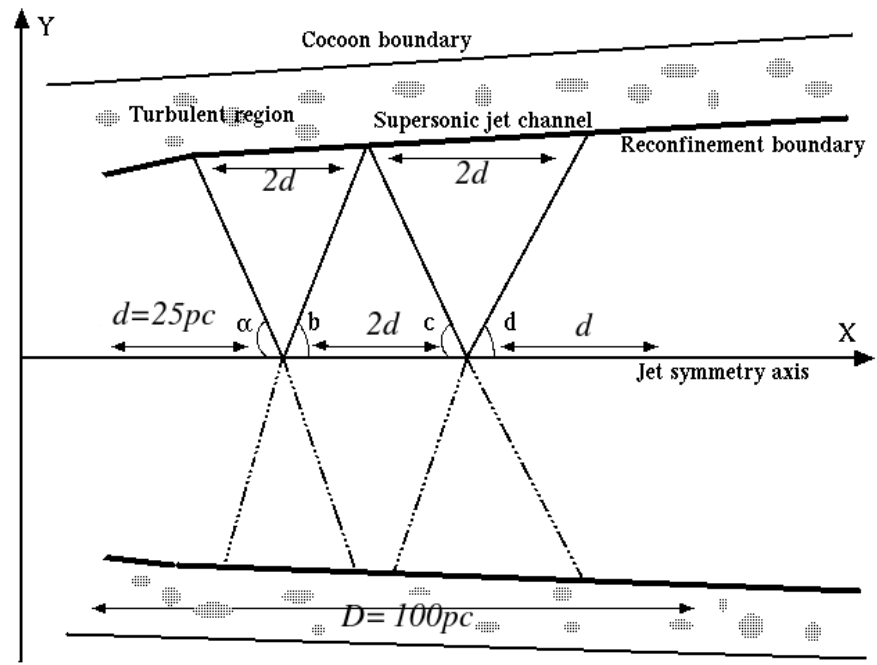

Fig. 5. Overall view of the proposed jet topology and conical shock geometries (not to scale) simulation framework. Here $d$ denotes a prefixed distance from the shock, $D$ is the total length of the acceleration area along the $x$-axis, which we assume here to be $100 \mathrm{pc}$.

(e.g. Earl et al. 1988; Stawarz \& Ostrowski 2002; Rieger \& Duffy 2006; Stawarz \& Petrosian 2008; Sahayanathan 2009), which ensures that there is no significant escape of particles off the jet reconfinement boundaries, which further more implies that this mechanism enhances the attainment of very high energies.

One would assume that every particle accelerating from one shock to another in the four-shock sequence will reach the next shock where it can be further accelerated. But, in a more realistic situation some particles found downstream of the forth shock, may have been accelerated in fewer that four shocks, therefore we allowed here a fixed escape probability $P_{\mathrm{e}}$ to be applied between the shocks during the acceleration process. This escape probability gives the fraction of particles of the downstream distribution of each shock that will not be further accelerated. These particles remain in the system and contribute directly to the downstream distribution of the forth shock. In the simulations shown, we chose $P_{\mathrm{e}}=0.3$ to simulate the decompression and shock extension between shocks in a repetitive sequence more realistically (e.g. Melrose \& Pope 1993). We note that $P_{\mathrm{e}}=1$ means that particles are produced by a single shock, while $P_{\mathrm{e}}=0$ shows that all particles are transported through the subsequent shocks.

For an extensive description of the numerical approach and particle kinematics refer to Meli \& Quenby (2003a,b), Meli et al. (2008), and the appendix.

\subsection{Single-oblique relativistic shock studies}

The Monte Carlo code and simulations of Meli \& Quenby (2003a,b, hereafter MQ03) and the follow-up systematic simulations of Meli et al. (2008, hereafter ME08) for single relativistic shocks is extended in this paper for multiple subluminal and superluminal shocks. Because we aim to consolidate the understanding of the results that will follow, we recapitulate and discuss here a selection of previous numerical studies based on the MQ03 Monte Carlo code for single relativistic shocks, and compare them with similar studies of Ellison \& Double (2004), Niemiec \& Ostrowski (2004), Baring (2004), and Summerlin \& Baring (2012). As we will see in Sect. 3.3, the behavior of the single relativistic shocks is continued into multiple relativistic shocks but with small deviations mostly due to the added factors of the decompression and relevant escape probability $P_{\mathrm{e}}$ (see Sect. 3.1).

MQ03 performed simulation studies for single parallel, oblique, and perpendicular relativistic shocks. It was examined how the relativistic shock speeds, shock obliquity (superluminal and subluminal) and diffusion operator affect the shape and spectral index of the particle spectra, the acceleration efficiency, the acceleration rates, and the particle angle distribution at shock transmission. Specifically, large angle scattering was assumed for the scattering operator such that $1 / \Gamma \ll \delta \theta \leq \pi$ and pitch angle diffusion such as $\delta \theta \leq 1 / \Gamma$. For a more in depth discussion on scattering see the appendix. Additionally, the assumed turbulence of the media in terms of mean free path was set to $\lambda=20 r_{\mathrm{g}}$. The supplemental Monte Carlo study of ME08 re-examined different shock conditions, investigating additional consequent applications to the cosmic-ray diffuse signal. In this work, systematic code runs were performed using a variety of shock inclinations (e.g. superluminal or subluminal) by decreasing the turbulence of the media to $\lambda=10 r_{\mathrm{g}}$ (compared with the MQ03, where $\lambda=20 r_{\mathrm{g}}$ ). Moreover, the pitch angle scattering was more relaxed than the MQ03 simulations such that $1 / \Gamma \leq \delta \theta \leq 10 / \Gamma$. We note that these values are also used in the present study.

The above relativistic shock acceleration simulation results showed a clear deviation of the spectral index value - compared to the nonrelativistic counterparts - that seemed to be dependent on three parameters: i) speed of the shock, ii) shock inclination, and iii) different scattering modes. The same observations are evident as well in the works of e.g. Ellison \& Double (2004), Baring (2004), Niemiec \& Ostrowski (2004), and Summerlin \& Baring (2012). In other words, Monte Carlo simulation studies independently showed that there is an absent "universal" powerlaw spectrum in relativistic shocks, and a variety of spectral indices depending either on shock inclinations, shock speeds, or scattering operators. In MQ03 and ME08 a high acceleration efficiency was found in terms of the maximum attained particle energy, which is evident in the results of the present study as well, see Sect. 3.3. It was also shown that both scattering operators (large angle scattering or pitch angle diffusion) yield significantly faster acceleration rates than the conventional nonrelativistic expression for the time constant, or alternatively for the time scale $r_{\mathrm{g}} / c$, an effect found also in e.g. Quenby \& Lieu (1989) and Ellison et al. (1995). Moreover, some structure was noticed in the particle spectrum that seemed to become enhanced for large angle scattering compared with pitch angle diffusion, rendering the idea of a simple power-law output spectrum uncertain, see also e.g. Ellison \& Double (2004), and Summerlin $\&$ Baring (2012) which agree with small quantitative differences due to different initial input parameter values of the simulations.

Specifically, the work of MQ03 directly agrees with that of Ellison \& Double (2004) who show that for the subluminal (quasi-parallel) shocks the large angle scattering exhibits distinctive structure superimposed on the basic power-law spectrum, which is largely absent in the pitch angle case. Furthermore these authors showed that in the relativistic case the lower the turbulence in terms of the mean free path $\eta=\lambda / r_{\mathrm{g}}$ the steeper the particle spectra, a feature that is also evident in Summerlin \& Baring (2012); and both agree with MQ03 work. Moreover, Ellison \& Double (2004) showed that the near-perpendicular shocks appear to give the steepest spectra compared to all other cases which directly agree with ME08. In Summerlin \& Baring (2012), in the pitch angle diffusion limit, the spectral index varies 
dramatically with obliquity as it is shown in MQ03 and ME08. We note again that the compared works identify the very same trends but with minor differences due to different initial parameter values in the simulations. Summarizing, we see that Ellison \& Double (2004) and Summerlin \& Baring (2012) agree with MQ03 and ME08, in the sense that as soon as the shock speed becomes comparable to the speed of light, the power-law index starts to depend strongly on shock obliquity and scattering operators, identifying steeper spectra for higher obliquity with an increased ratio $\lambda / r_{\mathrm{g}}$, but nevertheless flatter spectra than their nonrelativistic counterparts (see also Stecker et al. 2007).

Additionally, Niemec \& Ostrowski (2004) showed similar spectral slopes as in MQ03 and ME08. Although they used lower shock speeds $(\Gamma \leq 5)$ and a different particle diffusion approach in their Monte Carlo simulations (see also the appendix), their results confirm that the flatness or the irregular spectral distributions are dependent on inclination and scattering operators. Specifically, the superluminal (quasi-perpendicular) shocks yielded steeper spectra than all other cases which seemed to be less efficient in accelerating particles to very high energies, which directly agrees with the results of MQ03 and ME08.

In conclusion, is evident that our Monte Carlo studies for relativistic shock acceleration and those of different independent work groups agree well, which shows the consistency and reliability of the relativistic shock acceleration numerical studies, with small deviations due to slightly different approaches and different initial parametric values. MQ03 and ME08 specifically focused on the role of shock speed (i.e., $\Gamma$ ) and the consequent acquired maximum particle energy (i.e., in eV); two elements that are important in terms of radiative processes, which is emphasized again in the current work. Moreover, we will see in Sect. 3.3 how the behavior of the single relativistic shocks discussed above, continues to multiple shocks, albeit with small differences due to the added factors of the decompression and relevant escape probability $P_{\mathrm{e}}$, see Sect. 3.1.

\subsection{Multiple-oblique relativistic shocks}

In the nonrelativistic limit authors such as White (1985), Melrose \& Pope (1993), Schneider (1993), and Pope \& Melrose (1993), solved the problem of subsequent multiple shocks analytically calculating the spectrum as it is processed through a number of shocks, suppressing new injection, and considering adiabatic decompression. It has been shown analytically that the downstream distribution of particles after acceleration in a number of shocks alternating with decompression, establishes a power-law with flatter distributions in a single shock, e.g. Melrose \& Pope (1993) and Gieseler \& Jones (2000). For an infinite number of subsequent shocks with injection at each shock, the flattening of the spectrum (compared to a single shock) extends even to the highest energy particles, with a momentum dependence of $f(p) \propto p^{-3}$ (White 1985; Achterberg 1990). It is interesting to note here that this behavior seems equivalent to the shock produced by an infinite density jump (Drury 1983).

Here we investigate the acceleration of particles in relativistic superluminal and subluminal multiple shocks. Assuming blobs of hot plasma ejected by the black hole of an AGN can travel along its jet and pass through a sequence of shocks, as has observed for example in the case of BLac PKS 1510-089 (Marscher et al. 2010), we allowed a single initial injection of particles at the beginning of the acceleration of a fixed number of particles $\left(N_{i}=10^{4}\right.$ of an initial $\left.\gamma=\Gamma_{\text {sh }}+10\right)$, and used an initial shock Lorentz factor $(\Gamma=50)$ for the first conical pattern (which consists of two oblique shocks with the same apex and inclination angles $a$ and $b$ to the jet axis ( $x$ ), see Fig. 4).

For the second pair of shocks (with inclination angles $c$ and $d$ ) we used a $\Gamma=17$, which is physically justifiable because of the decompression-compression of the downstream plasma of the precedent shock pattern (i.e., a velocity compression ratio of 3).

As the particle properties are measured in the local plasma frame according to the relativistic jump conditions, the downstream properties for a shock become upstream properties for the subsequent one at a fixed $d=25 \mathrm{pc}$, see Fig. 5. We note that the shocks are simulated within a single simulation box, so some particles travel far upstream to a precedent shock and continue the acceleration. Nevertheless, due to the "beaming" effect of the particles in relativistic media (Achterberg et al. 2000; Meli \& Quenby 2003b; Meli et al. 2008) only few make it upstream to a previous shock.

As aforementioned, during the acceleration process a fixed probability of escape $P_{\mathrm{e}}$ between shocks due to decompression effects is also allowed (Melrose \& Pope 1993; Pope \& Melrose 1993), because it is assumed that every particle accelerating from one shock to another will always reach the next one and accelerate. It is expected that particles found downstream the 4th shock of our assumed sequence may have been accelerated in fewer than four shocks, thus a fixed escape probability $P_{\mathrm{e}}=0.3$ is allowed to be applied between the shocks during the acceleration process. In other words, the escape probability gives the fraction of particles of the downstream distribution of each shock that will not be accelerated more. These particles remain in the system and contribute directly to the downstream distribution of the 4th shock.

As discussed in Sect. 2, the shock inclination values may theoretically lie between perpendicular (superluminal), where a shock-drift acceleration mechanism occurs, and oblique (subluminal) ones, where diffusive acceleration takes place in the HT frame. One can see that in the relativistic case, half of the inclinations allowed are superluminal and the rest are subluminal, because of the $45^{\circ}$ transformation frame inclination limit, see Fig. 2 and Sect. 3.1.

It is shown by observations (e.g. Hughes et al. 1985; Cawthorne \& Wardle 1988) that when the jet contains an initially tangled magnetic field, plane shocks perpendicular to the jet axis produce polarization with an electric field perpendicular to the shock front (parallel to the shock normal), and hence parallel to the jet direction. Observationally this means, for a given parallel electric field to the jet axis the magnetic field is perpendicular to the shock normal, therefore particles trapped into the frozen-in plasma will become accelerated by repeatedly crossing the shock plane. One sees that this kind of topology serves as a large-scale analogon to the nondiffusive shock-drift acceleration mechanism we mentioned above, where a superluminal case applies. Nevertheless, as discussed in Sect. 2, theoretical and numerical studies show that the inclination of the oblique shocks inside a supersonic jet channel must have small angle values to the jet axis for a strong acceleration effect to occur. Therefore we assume that in the same manner, identical superluminal shocks are not very likely to occur during the acceleration phase of the AGN jet. Here we assume protons without losses, because we are interested in the spectra produced at the source, which is justifiable since the magnetic field in AGN jets can be weak (i.e., $B=10^{-4}$ Gauss).

Moreover, the temperature of an ambient photon field in a jet can be low, which imposes only a low loss factor for protons, for the considered scenario. In that sense, the initiation of secondary 

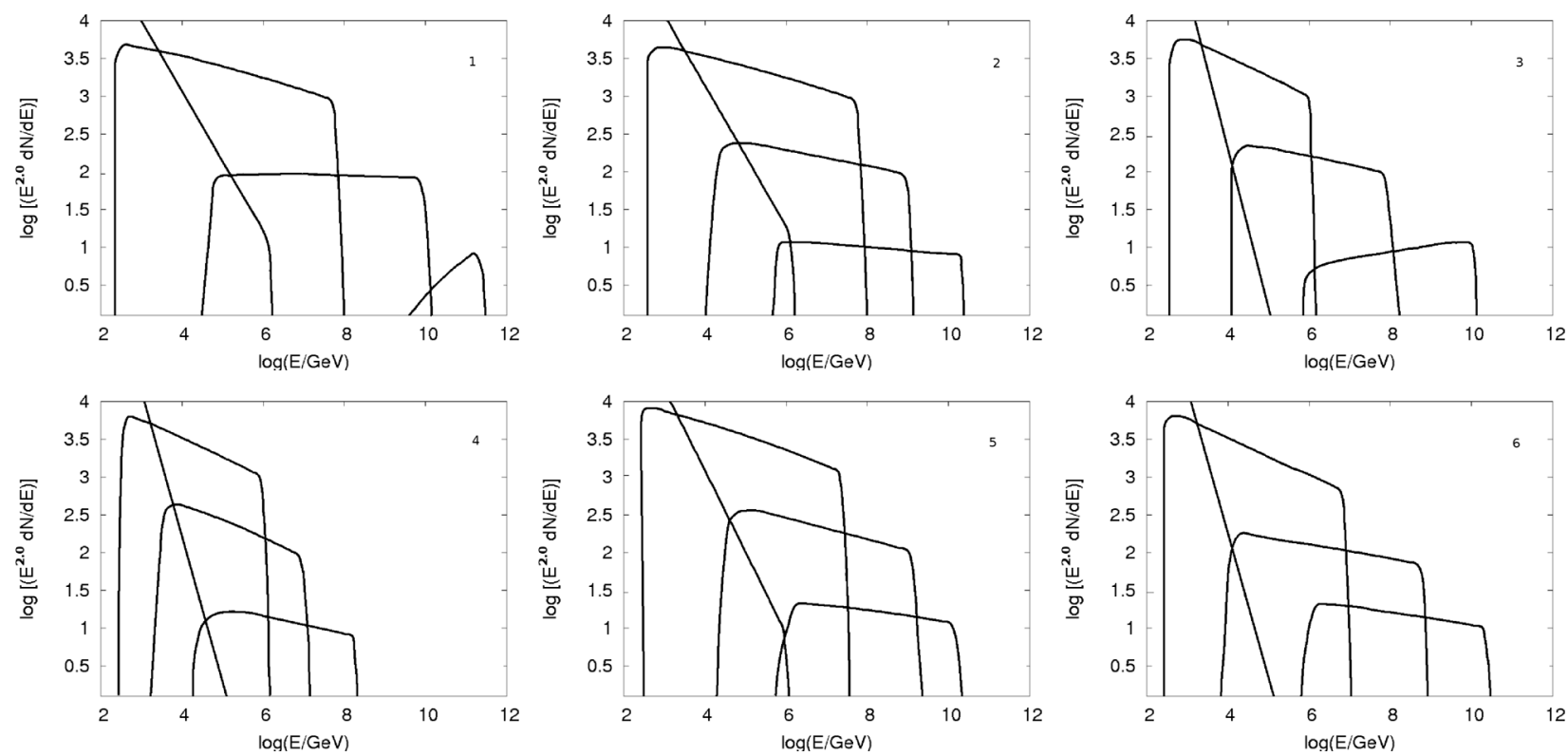

Fig. 6. Differential spectra (multiplied by $E^{2}$ ), calculated in the respective shock frame downstream, for sets of four oblique consecutive relativistic shocks. Here one sees developed flatter spectra (from left to right respectively) extending to very high energies with a depletion on the lower energies (analogy to Fig. 1). Panel 1: spectra for four consecutive subluminal shocks of inclination $\psi=45^{\circ}$ (corresponding to a half opening angle). Panel 2: spectra from two subluminal shocks and two superluminal shocks $\left(\psi=85^{\circ}\right)$. Panel 3: spectra for two superluminal and two subluminal shocks. Panel 4: spectra for four superluminal shocks all four for the same inclination $\psi=85^{\circ}$. Panel 5: spectra for a sequence of subluminal-superluminal-subluminal-superluminal shocks. Panel 6: spectra for a sequence of superluminal-subluminal-superluminal-subluminal shocks. See complimentary Table 1 . Spectra have been shifted vertically to allow a better comparison.

cascading, e.g., $p \gamma$ interaction, is minimal. This also agrees with field conditions far off the accretion disk-jet interaction area of the AGN (>3000 Schwarzschild-radii), see Markoff et al. (2005), and Becker \& Biermann (2009).

We performed a series of simulations for a range of shock Lorentz boost speed $\Gamma(10<\Gamma<50)$, finding quite similar spectral behaviors. Therefore, our chosen Lorentz shock speed is an exemplary case (because the velocity of plasma in AGN jets typically ranges between around 10 and 50 Lorentz factors). Six different sets of four consecutive oblique shocks were simulated for comparison purposes and their differential spectra are shown in Fig. 6. The spectra were recorded in the shock-rest frame downstream of each shock at a distance $d$, see Fig. 5. We note that we allowed two shock sets with identical inclination angles between them for simplicity and for direct comparisons, and because physically a decompression between shocks (e.g. Melrose \& Pope 1993) can restore the magnetic field to the initial upstream shock value and orientation. Nevertheless, there could be many other situations of non-identical inclinations between subsequent shocks, therefore we allowed this condition for other shock set profiles as well.

To facilitate our understanding, in Fig. 6 we depict the respective differential particle spectra by each shock in the same plot. The spectra were shifted vertically for better comparison. Specifically, in panel 1 we show spectra by four identical consecutive subluminal shocks, each with a half opening angle $\left(a, b, c, d=45^{\circ}\right)$ to the jet flow, equal to the actual inclination of the shock to the magnetic field, as we noted in Sect. 3.1, see Fig. 3. Panel 2 shows spectra for two subluminal $\left(a, b=45^{\circ}\right)$ and two superluminal shocks $\left(c, d=85^{\circ}\right)$. Panel 3 indicates spectra for two superluminal and two subluminal shocks, while panel 4 shows spectra from four identical superluminal shocks with the same inclination angles $\left(a, b, c, d=85^{\circ}\right)$.

Plots 5 and 6 show a set of subluminal-superluminalsubluminal-superluminal shocks and superluminal-subluminalsuperluminal-subluminal ones, respectively. Table 1 is complimentary to Fig. 6, showing values for the inclination of the shocks in each set, the spectral indices, and the highest attained energies by each shock. Since we are dealing with relativistic shocks, the pitch angle scattering was chosen to be $1 / \Gamma \leq \delta \theta \leq 10 / \Gamma$, with media a turbulence of $\lambda=10 r_{\mathrm{g}}$, based on the analytical description given in the appendix.

Firstly, by inspecting panel 1 (Fig. 6), one sees the highest particle energy reached an impressive $\sim 10^{11} \mathrm{GeV}$. In panel 4 , the case of four consecutive superluminal shocks is shown which is the least efficient, with a highest attained particle energy of a modest $\sim 10^{8} \mathrm{GeV}$. Physically, the four identical superluminal shocks would in fact require a repetitive set of oblique shocks with very large opening angles $\left(a, b, c, d \approx 90^{\circ}\right)$, meaning a continuous decelerating flow for a distance of about $100 \mathrm{pc}$, but as we discussed in Sect. 2, this is not realistic.

Second, one notices that (i) the spectra by each shock in every shock-set become gradually flatter, following on one hand the findings of single relativistic shocks (e.g. Ellison et al. 1995; Stecker 2007; Meli et al. 2008), and on the other hand the trends reported in White (1985), Melrose \& Pope (1993), Schneider (1993), and Gieseler \& Jones (2000) for multiple shocks. Specifically among others, the later work noted that the flat particle spectra from multiple shocks is due to the obliquity (identical or not) of the shocks, a result that agrees with the present study. (ii) The flatness of the spectra is more evident for the consecutive subluminal shocks than for the superluminal ones, following, as expected, the individual shock effects we discussed in Sect. 3.2. It is important to note that superluminal shocks result in steeper spectra than subluminal shocks, but all (relativistic, single or multiple) result in flatter energy distributions than their nonrelativistic counterparts.

Since a fixed number of particles was injected once and accelerated through more than one shock, this means in other words a gradual decreased flux at lower energies and a consequent "extension" of the energy distributions to higher energies. For example, for the flattest spectra (panel 1, Fig. 6) one sees a 
Table 1. Shock inclinations, spectral indices, and highest energies attained for the six cases of four shock combinations (complimentary to the spectra shown in Fig. 6).

\begin{tabular}{lcc}
\hline \hline Shock inclination & Spectral index & $E_{\max }[\mathrm{GeV}]$ \\
\hline $45^{\circ}$ subluminal & -2.7 & $10^{6}$ \\
$45^{\circ}$ subluminal & -2.2 & $10^{8}$ \\
$45^{\circ}$ subluminal & -2.0 & $10^{10}$ \\
$45^{\circ}$ subluminal & -1.4 & $10^{11.5}$ \\
\hline
\end{tabular}

Panel a

\begin{tabular}{lcc}
\hline \hline Shock inclination & Spectral index & $E_{\max }[\mathrm{GeV}]$ \\
\hline $45^{\circ}$ subluminal & -2.7 & $10^{6}$ \\
$45^{\circ}$ subluminal & -2.2 & $10^{8}$ \\
$85^{\circ}$ superluminal & -2.2 & $10^{9}$ \\
$85^{\circ}$ superluminal & -2.1 & $10^{10}$ \\
\hline
\end{tabular}

Panel b

\begin{tabular}{lcc}
\hline \hline Shock inclination & Spectral index & $E_{\max }[\mathrm{GeV}]$ \\
\hline $85^{\circ}$ superluminal & -2.8 & $10^{5}$ \\
$85^{\circ}$ superluminal & -2.4 & $10^{6}$ \\
$45^{\circ}$ subluminal & -2.1 & $10^{8}$ \\
$45^{\circ}$ subluminal & -1.6 & $10^{10}$ \\
\hline
\end{tabular}

Panel c

\begin{tabular}{lcc}
\hline \hline Shock inclination & Spectral index & $E_{\max }[\mathrm{GeV}]$ \\
\hline $85^{\circ}$ superluminal & -2.8 & $10^{5}$ \\
$85^{\circ}$ superluminal & -2.4 & $10^{6}$ \\
$85^{\circ}$ superluminal & -2.4 & $10^{7}$ \\
$85^{\circ}$ superluminal & -2.3 & $10^{8}$ \\
\hline
\end{tabular}

Panel d

\begin{tabular}{lcc}
\hline \hline Shock inclination & Spectral index & $E_{\max }[\mathrm{GeV}]$ \\
\hline $45^{\circ}$ subluminal & -2.7 & $10^{6}$ \\
$85^{\circ}$ superluminal & -2.5 & $10^{8}$ \\
$45^{\circ}$ subluminal & -2.3 & $10^{9}$ \\
$85^{\circ}$ superluminal & -2.1 & $10^{10}$ \\
\hline
\end{tabular}

Panel e

\begin{tabular}{lcc}
\hline \hline Shock inclination & Spectral index & $E_{\max }[\mathrm{GeV}]$ \\
\hline $85^{\circ}$ superluminal & -2.8 & $10^{5}$ \\
$45^{\circ}$ subluminal & -2.4 & $10^{7}$ \\
$85^{\circ}$ superluminal & -2.2 & $10^{9}$ \\
$45^{\circ}$ subluminal & -2.1 & $10^{10}$ \\
\hline Panel f & &
\end{tabular}

Panel f

Notes. Each part of the table corresponds to each panel of Fig. 6 .

change in spectral fluxes by as much as six orders of magnitude. The same trend is seen in all other cases. This is an important result in terms of source power interpretation, which we discuss below.

In more detail:

1. The first two shocks in all shock sequences range between spectral index $(\sigma)$ values of -2.7 to -2.2 for subluminal shocks, and -2.8 to -2.4 for superluminal ones.

2. The sequence of four identical subluminal shocks generates the flattest spectra of all other cases, with the flattest value reaching $\sigma$ at -1.4 , and the highest achieved particle energy at $\sim 10^{11} \mathrm{GeV}$.

3. Four consecutive identical superluminal shocks generate the steepest spectra with $\sigma \sim-2.3$, and the lowest particle energy attained of $10^{8} \mathrm{GeV}$.
4. In all spectra, one sees a flux depletion in lower energies and an additional gradual flatness of the slopes, with an extension of the spectra to high energies. This is because fewer and fewer particles participate in the acceleration at the highest of energies, but this is balanced by the properties of relativistic shock acceleration, such as acceleration speed-up (see Meli \& Quenby 2003b) and relativistic subluminal shock acceleration efficiency. We note here that our spectra look very similar to those of Melrose \& Crouch (1997), although these authors treated nonrelativistic shocks.

5. In all six shock sequences, two consecutive subluminal shocks flatten the spectra by 0.5 . On the other hand, their superluminal counterparts remain steeper with only a low spectral index deviation value of 0.1 . In the first case (see Table 1.a) of the four identical subluminal shocks the spectra flatten by 1.3 with a similar behavior for case 3 (see Table 1.c) of two superluminal and two subluminal shocks, with an overall flattening of 1.2. For the rest of the cases we see an overall flattening by $0.6,0.5,0.6$, and 0.7 respectively for cases 2, 4, 5, and 6. Because the pitch angle diffusion scatter is fixed, the variations of the slopes are connected to the individual subluminal or superluminal shock properties discussed in Sect. 3.2, but also on the conditions prescribed for multiple acceleration, i.e., an escape probability $P_{\mathrm{e}}$ factor between consecutive shocks, identical inclinations of shocks to the jet flow, i.e., superluminal or subluminal shocks, or pair alteration between the two inclinations, etc.

Examining our model results in an astrophysical context, one derives that the first shock from a repetitive sequence in an AGN jet could establish a low-energy power-law spectrum with a spectral index of $\sim 2.7$, while the following shocks of the sequence push the particle spectrum up in energy. One observes gradual flatter energy distributions, but most importantly the flux depletion at lower energies, forming characteristic starved spectra. This means that with a single injection of low-energy particles through a set of oblique shocks, one can achieve very high energies through acceleration in a set of separate starved spectra which over a superimposition could give flatter spectra in very high energies, solving in that way the puzzling apparent source power problem of AGN fed into the very high energy cosmic-rays.

As we discussed in Sect. 1, the best-fit injection power-law index value used in the models of de Marco \& Stanev (2005), and Berezinsky et al. (2006) is between -2.4 and -2.7 , for particle energies above $\sim 10^{9} \mathrm{GeV}$. Specifically, in that work theoretical comparisons were made with observation data by the HiRes (Sokolsky et al. 2009) and Auger experiments (Abraham et al. 2007, 2010a, 2010b). Their model assumed a number of sources homogeneously distributed throughout the universe. The particles accelerated by these sources were then let to propagate. The initial spectral index necessary to reproduce the observations, assuming that the particles were protons, was found to be within the values of -2.4 to -2.7 , which is quite close to many cases shown in this work.

We also showed that consecutive subluminal shocks result in very flat starved spectra, up to UHECR values. To fit the required spectra of de Marco \& Stanev (2005) and Berezinsky et al. (2006), it seems obvious that if one assumed protons accelerated at the source, there must be some dense photon field nearby where one could achieve steeper spectra via proton synchrotron losses, but on the other hand this would compromise the energies reached in the primary accelerated population. Thus it could mean that after these particles interact, they may reaccelerate again, having their spectra pushed up in energy into 
the UHECR energy regime, retaining their spectral index before they start to propagate. The re-acceleration could happen in a nearby source like CenA through relativistic shock acceleration, where these particle spectra could be pushed up in energy before reaching Earth's observatories, as Biermann \& de Souza (2011) showed.

Alternatively, assuming there could be a single source of very high energy particles within $d \simeq 50 \mathrm{Mpc}$ (note that CenA is within 3-5 Mpc and M 87 is within 16-17 Mpc), given our results, one could require additional losses to steepen the spectra accordingly, which could be feasible if the population of particles were a mixture of protons and heavier nuclei (e.g., Fe), see Aloisio et al. (2012), and Biermann \& de Souza (2012). It is interesting to note here that the distance of either CenA or M87 and the interaction of protons with the microwave background is not quite as important, see Greisen (1966), and Zatsepin \& Kuzmin (1966), except for the highest energies.

On the other hand, if there were more than one source of very high-energy cosmic-rays (above $\sim 10^{9} \mathrm{GeV}$ ) within $50 \mathrm{Mpc}$ (which we do not see yet), one would require a superposition of their spectra, as an integrated signal over the different shock profiles shown in this work, due to energy problems we described in Sect. 1, to satisfy these calculations. It is interesting to note here that of course there maybe many very high energy particle extragalactic sources, but it is hard to believe that they are all be sources of heavy nuclei only.

Apparently, our present study seems to agree with the latest Auger data measurements, in connection with a proton composition in lower energies and probably a heavier composition imprint above $\sim 10^{10} \mathrm{GeV}$. It is important to note that our model results in spectra which can reach a maximum of $\sim 10^{11} \mathrm{GeV}$ for only one case, while the rest reach energies below $10^{10} \mathrm{GeV}$, which justifies the first half of the UHECR energy scale. For higher attained energies (up to $10^{12} \mathrm{GeV}$ ), higher Lorentz gamma shock factors than $\sim 50$ may be needed as was shown in Meli et al. (2008), but for AGN jet velocities this seems unlikely, except for some few cases, e.g., 3C 279. We furthermore note that the present results are fully consistent with the observations of Lister et al. (2011).

Our acceleration model could explain the variability of flat or inverted gamma-ray spectra observed in high-redshifted flaring extragalactic sources (e.g. 3C 279; Albert et al. 2008). These spectra may originate from particles accelerating and radiating from within a region of the topology we investigated, resulting in the characteristic starved spectra shown in this work.

It seems possible that the population of UHECR consists not only of protons for the whole energy spectrum examined here, but it can be a mixture of heavy nuclei and protons. If this is the case, it seems logical that a mixture of particles accelerating in a shock sequence as the one described here could yield steeper spectra, because heavy nuclei suffer photo-disintegration or fragmentation for the highest energy spectral end. This condition could change the spectral signatures considerably. We would like to examine this aspect in more detail in the near future.

\section{Conclusions}

We performed Monte Carlo simulations allowing diffusive particle acceleration at relativistic multiple-oblique shocks as an application to multiple shocks observed in various AGN relativistic jets. We based our work on the reconfinement mechanism in jets and the incomplete Comptonization effect, and we injected test-particles once upstream the first shock of a shock sequence, letting them accelerate through different sets of subluminal and superluminal shocks.

Specifically, we studied an exemplary case for six possible combinations of a set of four consecutive relativistic oblique shocks, with an aim to improve our understanding on the acceleration efficiency and the properties of the produced particle spectra. In principle, our investigation was initiated by the fact that particle energies with $\geq 10^{18.5} \mathrm{eV}$ imply much more total source power. We showed that when flat and starved particle spectra are attainable, the puzzling extra power to be provided for UHECRs does not seem necessary. By injecting particles once toward a sequence of shocks, one could obtain a final particle spectrum extending up to very high energies with depletion at lower energies with a smaller number of low-energy particles. We numerically showed that the first shock from the multipleshock sequence establishes a low-energy power-law spectrum with $\sim E^{-2.7}$, while the consecutive shocks push the particle spectrum up in energy with flatter distributions, leaving a flux deficiency at low energies.

We showed that for two or more shocks the spectrum becomes flatter than -2.2. Especially when only identical subluminal shocks are involved, the spectra are the flattest and the highest particle energy of a spectacular $10^{11} \mathrm{eV}$ is attained, which also confirms the expected high efficiency of subluminal shocks shown previously in Meli \& Quenby (2003b) and Meli et al. (2008). In contrast, when only superluminal shocks are present, they render the spectra steeper and the acceleration less efficient.

Within our model, other particles except protons (e.g. electrons, heavy nuclei) would naturally suffer losses in addition to all the effects from oblique shocks that could accentuate the sharpness of the final spectrum, as we discussed above, which is of interest to our astrophysical interpretation.

Consequently, considering the resutls of de Marco \& Stanev (2005), and Berezinsky et al. (2006), which require an injected source spectrum for UHECRs between $E^{-2.4}$ and $E^{-2.7}$ before propagation, our model can explain this on the basis of either a single source such as CenA that accelerates a composite population of protons and heavy nuclei that are initially flat at source (justifying source power requirements) with additional propagation energy losses and a significant steepening, or due to the superposition of several sources, all of which end their acceleration shock sequence with two or more relativistic subluminal shocks with starved or inverted flat spectra (also justifying source power requirements).

Moreover, with the current model one may explain irregular or very flat gamma-ray spectra by various flaring high redshifted extragalactic sources. Specifically, inverted spectra may be required to comprehend the tendency to detect TeV sources at redshifts such as 0.5 (3C 279; Albert et al. 2008); sources with normal spectra would be undetectable. This remains to be examined in more detail in a follow-up work. Intensive future observations may yet test or reveal one or more of the above predictions.

Acknowledgements. We would like to thank the anonymous referee for the constructive comments on this work and we acknowledge fruitful discussions with Prajval Shastri (Indian Institute of Astrophysics, Bangalore). Support for work with PLB has come through the AUGER membership.

\section{Appendix A: Turbulence and scattering}

In standard kinetic theory the spatial diffusion coefficients $\kappa_{\|}$ and $\kappa_{\perp}$ are related to the formula $\kappa_{\perp}=\kappa_{\|} \cdot\left(1+\left(\lambda / r_{\mathrm{g}}\right)^{2}\right)^{-1}$ (Ellison et al. 1995), where $\lambda$ is particle's m.f.p. The ratio $\lambda / r_{\mathrm{g}}$ represents the level of media turbulence, where $r_{\mathrm{g}}$ is the Larmor radius in the plane of gyration. In parallel shocks, where the magnetic 
field B field is directed along the shock normal (i.e., $\psi=0^{\circ}$ ), the ratio $\lambda / r_{\mathrm{g}}$ has a limited impact on the produced energy spectrum, which in principal determines the diffusive spatial scale normal to the shock. Nevertheless, in oblique relativistic shocks $\left(\psi>0^{\circ}\right)$, the diffusive transport of particles across the shock, or in other words across the field, becomes critical in retaining them into the acceleration process. Therefore, the interplay between the field angle and the value of the ratio $\lambda / r_{\mathrm{g}}$ determines the spectral index of the particle distribution (see also Ellison \& Double 2004).

Because a shock acceleration process implies that this energy gain rate scales as the gyrofrequency $c / r_{\mathrm{g}}$, i.e., is proportional to the particles energy/momentum, in our work, a simple representation for the effect of the turbulence is to assume that the particle scatters $\delta \theta=M / \Gamma$ every $\lambda=N r_{\mathrm{g}}$ where $M$ and $N$ chosen numbers. In the well-known Bohm limit, $\lambda / r_{\mathrm{g}}=1$, where the field fluctuations satisfy $\delta B / B \sim 1$. Interplanetary particle propagation studies and gyroresonance theory suggest $\lambda$ to be a number of particle gyroradii $r_{\mathrm{g}}$, that is, $\lambda \geq 10 r_{\mathrm{g}}$. Moreover, in the shock normal, or in the $x$ direction, the diffusion coefficient can be written as $\kappa_{\|}=\lambda u / 3$, when no cross-field diffusion occurs. When cross-field diffusion is present due to increased turbulence, then $\kappa=\kappa_{\|} \cos ^{2} \psi+\kappa_{\perp} \sin ^{2} \psi$ (Jokipii 1987), where $\psi$ denotes the inclination of the shock normal to the magnetic field. For simplicity however it is generally assumed throughout the literature that $\kappa_{\|} \gg \kappa_{\perp}$, therefore a guiding center approximation can be applied more accurately .

Additionally, it is assumed that $\lambda$ is proportional to a power of the particle's momentum $p$ (Giacalone et al. 1992), which is assumed to scale as the particle gyroradius $r_{\mathrm{g}}$, i.e., $\lambda=N r_{\mathrm{g}} \propto$ $p$. When one reduces $\lambda / r_{\mathrm{g}}$ turbulence is increasing by allowing a very small degree cross-field diffusion. This in turn may in general steepen the spectrum. For values of $\lambda / r_{\mathrm{g}}<10$ one may assume that qualitatively the shock behaves more like a parallel subluminal shock in terms of its diffusive character.

The so so-called large angle scattering is when particles scatter as $1 / \Gamma \ll \delta \theta \leq \pi$, while in all other cases one has a pitch angle diffusion, with extreme cases as $\delta \theta \leq 1 / \Gamma$ (where $\Gamma$ denotes the Lorentz factor of the flow). We mention here that in Gallant et al. (1999) it was demonstrated for relativistic shocks analytically that particles entering the upstream region in a direction nearly normal to the shock can only experience pitch angle diffusion of values $\delta \theta \leq 1 / \Gamma$, with $\delta \theta$ measured in the upstream fluid frame for scattering in a uniform field or a randomly oriented set of uniform field cells. This condition arises because particles attempting to penetrate upstream from the shock are swept back into the shock before they can scatter far from it. But this is not entirely true, since Gallant et al. (1999) considered only the parallel shock case (see also Ellison et al. 1995; Baring 2009). It is obvious that for oblique shocks the kinematics involving particle diffusion, turbulence ratio, and the specific shock inclinations play a critical role that affects the acceleration efficiency and particle spectra, as we will show below. This would be especially relevant if the angle of the shock relative to the overall jet axis were to approach $1 / \Gamma$.

It is true that in relativistic shocks the achieved energy constraint of particles is largely dependent on the kinematic competition between upstream particle flow and the relativistic approach of the shock front, on the other hand though it is not critically dependent on the exact magnitude of the pitch angle scattering, as was extensively discussed in e.g., Meli et al. (2008). One can allow pitch angle scattering greater than the extreme aforementioned case of $\delta \theta \leq 1 / \Gamma$. Furthermore, because the downstream scattering is relevant to particle loss, it seems reasonable to use higher values of $\delta \theta$ within the pitch angle scattering model.

A transverse field perturbation changes the pitch angle in a quasi-linear theory (Kennel 1966) by

$\delta \theta=\omega \frac{b}{B} \delta t$

in a time $\delta t$ due to a perpendicular perturbation $b$ to the mean field $B$ with cyclotron angular frequency $\omega=e B / \gamma m_{\circ} c$. The particle moves in near gyro-resonance with the wave in $b$. A pitch angle diffusion coefficient can then be derived

$D_{\theta}=\frac{\delta \theta^{2}}{\delta t}=\frac{\omega^{2}}{u_{\|}} \frac{P(k)}{B^{2}}$

where $P(k)=P_{\circ} k^{\mathrm{s}}$ is the power spectral density of $b$ at gyroresonance wave number $k=\omega / u_{\|}$. A particle then diffuses in pitch by a finite amount $\delta \theta$ during $\delta t$ given by

$\delta \theta^{2}=2 D_{\theta} \delta t$.

Waves of wave number $k$, fulfil the gyroresonance condition, $k=$ $\omega / u_{\|}$that cause the scattering of particles satisfying $\omega r_{\mathrm{g}}=u_{\perp}$. In the simulations we also allowed the particles with pitch angle chosen at random to lie in the range of $1 / \Gamma \leq \delta \theta \leq 10 / \Gamma$. On average, a particle scatters $5 / \Gamma$ after a time $10 \sqrt{3} r_{\mathrm{g}} / c$ so that

$\delta \theta^{2}=\frac{25}{\Gamma^{2}}=\frac{2 \omega^{2}}{u_{\|} B^{2}} P_{\circ}(k) \frac{\omega^{\mathrm{s}}}{u_{\|}^{\mathrm{s}}} \frac{10 \sqrt{3} u_{\perp}}{c \omega}$.

To choose $\delta \theta$ independent of particle $\gamma$ that is of $\omega$, we require $s=-1$ for the spectral slope. Then we obtain a power spectrum relative to the mean field power

$\frac{P(k)}{B^{2}}=\frac{5}{4 \sqrt{2} \Gamma^{2}} k^{-1}$.

If resonating waves are present to scatter particles of between $\gamma=300$ and $\gamma=10^{12}$, the total fractional power in the turbulence is $1.4 / \Gamma^{2}$. Hence, the chosen pitch angle scattering model corresponds to a mild turbulence situation, accordingly, we chose here $N=10$, that is, $\lambda=10 r_{\mathrm{g}}$.

Because the quasi-linear theory for wave particle interactions is known to be an inexact approximation, the power spectrum we presented must also be an approximation to the scattering model we employ. The choice of a fixed factor $N=10$ in the relation between $\lambda$ and $r_{\mathrm{g}}$ acknowledges this inexactness. This discussion was presented to provide a link with the work of others, especially Niemec \& Ostrowski (2004), who represented field fluctuations by employing predefined wave spectra in their studies.

\section{References}

Abbasi, R. U., Abdou, Y., Abu-Zayyad, T., et al. (IceCube Collaboration) 2012, Nature, 484, 351

Abdo, A. A., Ackermann, M., Ajello, M., et al. (Fermi-LAT Coll.) 2010, Science, published online, 10.1126/science. 1184656

Abraham, J., Ackermann, M., Ajello, M., et al. (The Pierre Auger Collaboration) 2007, Science, 318, 938

Abraham, J., Abreu, P., Aglietta, M., et al. (The Pierre Auger Collaboration) 2010a, Phys. Rev. Lett., 104, id. 091101

Abraham, J., Abreu, P., Aglietta, M., et al. (The Pierre Auger Collaboration) 2010b, Phys. Lett. B, 685, 239

Achterberg, A. 1990, A\&A, 231, 251

Achterberg, A. 2000, IAU Symp., 195, 291

Agudo, I., Gomez, J.-L., Mart, J.-M., et al. 2001, ApSS, 276, 293 
Albert, J., Aliu, E., Anderhub, H., et al. (The MAGIC Collaboration) 2008, Science, 320, 1752

Aller, H. D., Aller, M. F., \& Hughes, P. A., 1985, ApJ, 298, 296

Aloisio, R., Berezinsky, V., \&Gazizov, A. 2012, J. Phys. Conf. Ser., 337, 012042 Ampleford, D. J., Jennings, C. A., Lebedev, S. V., et al. 2008, APS, APR8HE013 Arnold, C. N., \& Arnett, W. D. 1986, ApJ, 305, L57

Asada, K., Inoue, M., Nakamura, M., Kameno, S., \& Nagai, H. 2008, ApJ, 682, 798

Axford, W. I., Leer, E., \& Skadron, G. 1997, ICRC, 11, 132

Baring, M. G. 2004, Nucl. Phys. B, 136198

Baring, M. G. 2009, AIP Conf., 1133, 294

Becker, J. K., \& Biermann, P. L. 2009, Astropart. Phys., 31, 138

Begelman, M. C., \& Kirk, J. G. 1990, ApJ, 353, 66

Bell, A. R. 1978a, MNRAS, 182, 147

Bell, A. R. 1978b, MNRAS, 182, 443

Berezinsky, V., Gazizov, A., \& Grigorieva, S. 2006, Phys. Rev. D, 74, 043005

Blandford, R. 1993, in Relativistic Astrophysics and Particle Cosmology, eds. C. W. Akerlof, \& M. A. Srednicki, Annals of the New York Academy of Sciences (New York: NY Academy of Sciences), 688, 311

Blandford, R. D., \& Znajek, R. L. 1977, MNRAS, 179, 433

Blandford, R. D., \& Ostriker, J. P. 1978, ApJ, 221, L29

Biermann, P. L. 1993, A\&A, 271, 649

Biermann, P. L. 1994, High Energy Astrophysics, ed. J. M. Matthews (Singapore: World Scientific), 217

Biermann, P. L., \& Cassinelli, J. P. 1993, A\&A, 277, 691

Biermann, P. L., \& de Souza, V. 2012, ApJ, 746, 72

Biermann, P. L., \& Strittmatter, P. A. 1987, ApJ, 322, 643

Biermann, P. L., Becker, J. K., Meli, A., et al. 2009, Phys. Rev. Lett., 103, 1101

Biermann, P. L., Becker, J. K., Caceres, G., et al. 2010a, ApJ, 710, L53

Biermann, P. L., Becker, J. K., Dreyer, J., et al. 2010a, ApJ, 7, 184

Bowman, M., Leahy, J. P., Komissarov, S. S. 1996, MNRAS, 279, 899

Bridle A. H., \& Perley R. A. 1984, ARA\&A, 22, 319

Britzen, S., Vermeulen, R. C., Campbell, R. M., et al. 2008, A\&A, 484, 119

Brown, E., Lyutikov, M., \& Kharb, P. 2009, APS, E1005

Cawthorne, T. V. 2006, MNRAS, 367, 851

Cawthorne, T. V., \& Cobb, W. K. 1990, ApJ, 350, 536

Cawthorne, T. V., \& Wardle, J. F. C. 1988, ApJ, 332, 696

Cheung, C. C., Harris, D. E., \& Stawarz, L. 2007, ApJ, 663, L65

Clarke, D. A. 1996, AAS, 189, 4004

Darwin, C. 1953, Proc. Cambrige Philosophical Society, 49, 342

de Hoffmann, F., \& Teller, E. 1950, Phys. Rev., 80, 692

de Marco, D., \& Stanev, T. 2005, Phys. Rev. D, 72, 1301

Dingus, B. L. 1995, Astron. Space Sci., 231, 187

Drury, L. O. 1983, Rep. Prog. Phys., 46, 973

Duncan, G. C., \& Hughes, P. A. 1994, ApJ, 436, L119

Earl, J. A., Jokipii, J. R., \& Morfill, G. 1988, ApJ, 331, L91

Ellison, D. C., \& Double, G. P. 2004, Astropart. Phys., 22, 323

Ellison, D. C., Baring, M. G., \& Jones, F. C. 1995, ApJ, 453, 873

Falcke, H., \& Biermann, P. L. 1995, A\&A, 293, 665

Falcke, H., Malkan, M. A., \& Biermann, P. L. 1995, A\&A, 298, 375

Fermi, E. 1949, Phys. Rev., 75, 1169

Fermi, E. 1954, ApJ, 119, 1

Fukue, J., \& Okada, R. 1990, PASJ, 42, 249

Gabuzda, D. C., Murray, E., \& Cronin, P. 2004, MNRAS, 351, L89

Gallant, Y. A., Achterberg, A., \& Kirk, J. G. 1999, A\&AS, 138, 549

Giacalone, J., Burgess, D., Schwartz, S. J., \& Ellison, D. C. 1992, Geophys. Rev. Lett., 19, 433

Gieseler, U. D. J., \& Jones, T. W. 2000, A\&A, 357, 1133

Ginzburg, V. L., \& Syrovatskii, S. I. 1963, Sov. Astron., 7, 357

Gopal-Krishna, B. P. L., Souza de V., \& Wiita, P. J. 2010, ApJ, 720, L155

Greisen, K. 1966, Phys. Rev. Lett., 16, 748

Hardcastle, M. J., Kraft, R. P., Sivakoff, G., et al. 2007, ApJ, 670, L81

Hillas, A. M. 1984, ARA\&A, 22, 425

Hudson, P. D. 1965, MNRAS, 131, 23

Hughes, P. A., Aller, H. D., \& Aller, M. F. 1985, ApJ, 298, 301

Jokipii, J. R. 1987, ApJ, 313, 842

Jones, T. W., Tregillis, I. L., \& Ryu, D. 2011, ASP Conf., 250, 324

Jorstad, S. G., Marscher, A. P., Stevens, J. A., et al. 2007, AJ, 134, 799

Kardashev, N. S. 1962, Sov. Astron., 6, 317

Katz, J. I. 1976, ApJ, 206, 910

Kennel, C. F., \& Petscheck, H. E. 1966, J. Geophys. Res., 71, 1
Keppens, R., Meliani, Z., van der Holst, B., \& Casse, F. 2008, A\&A, 486, 663

Kharb, P., Shastri, P., \& Gabuzda, D. C. 2005, ApJ, 632, L69

Kirk, J. G., \& Heavens, A. F. 1989, MNRAS, 239, 995

Kobayakawa, K., Honda, Y. S., \& Samura, T. 2002, Phys. Rev. D, 66, 3004

Konigl, A. 1980, Phys. Fluids, 23, 1083

Korchakov, A. A., \& Syrovat-Skii, S. I. 1962, Sov. Astron., 5, 678

Krymskii, G. F. 1977, Dokl. Acad. Nauk. Akademiia Nauk SSSR, Doklady, 234, 1306

Lagage, P. O., \& Cesarsky, C. J. 1983, A\&A, 125, 249

Lebedev, S. V., Ciardi, A., Ampleford, D. J., et al. 2005, Plasma Phys. Controlled Fusion, 47, 465

Leighton, R. B. 1959, Book Principles of Modern Physics (NY.: MCGraw-Hill Book Company, Inc.)

Lind, K. R., \& Blandford, R. D. 1985, ApJ, 295, 358

Lister, M. L., Aller, M., Aller, H., et al. (MOJAVE Collaboration) 2011, ApJ, 742,27

Lovelace, R. V. E. 1976, Nature, 262, 649

Machalski, J., Chyży, K. T., Stawarz, Ł., \& Kozieł, D. 2007, A\&A, 462, 43

Markoff, S., Nowak, M. A., \& Wilms, J. 2005, ApJ, 635, 1203

Marscher, A. P., Jorstad, S. G., D’Arcangelo, F. D., et al. 2008, Nature, 452, 966

Marscher, A. P., Jorstad, S. G., Larionov, V. M., et al. 2010, ApJ, 710, L126

Marti, J. M., Mueller, E., \& Ibanez, J. M. 1994, A\&A, 281, L9

Marti, J. M., Muller, E., Font, J. A., \& Ibanez, J. M. 1995, ApJ, 448, L105

Massaglia, S., Bodo, G., Ferrari, A., \& Rossi, P. 1996, Lect. Not. Phys., 471, 275

Meli, A. 2003, Ph.D. Thesis, Imperial College London, UK

Meli, A., \& Quenby, J. J. 2003a, Astroart. Phys., 19, 637

Meli, A., \& Quenby, J. J. 2003b, Astroart. Phys., 19, 649

Meli, A., \& Biermann, P. L. 2006, A\&A, 454, 687

Meli, A., Becker, J. K., \& Quenby, J. J. 2008, A\&A, 492, 323

Melrose, D. B., \& Pope, M. H. 1993, PASA, 10, 222

Melrose, D. B., \& Crouch, A. 1997, Astron. Soc. Aust., 14, 251

Nakamura, M., Garofalo, D. A., \& Meier, D. L. 2010, A\&AS, 215, 434.15

Nalewajko, K. 2009, MNRAS, 395, 524

Nartallo, R., Gear, W. K., Murray, A. G., Robson, E. I., \& Hough, J. H. 1998, MNRAS, 297, 667

Niemiec, J., \& Ostrowski, M. 2004, ApJ, 610, 851

O’Neill, S. M., Jones, T. W., Tregillis, I. L., \& Ryu, D. 2006, Astron. Nachtr., 327,535

Ostrowski, M. 2008 [arXiv : 0801. 1339]

Parker, E. N. 1953, AJ, 58, 46

Parker, E. N. 1958, ApJ, 128, 664

Pope, M. H., \& Melrose, D. B. 1994, PASA, 11, 175

Quenby, J. J., \& Lieu, R. 1989, Nature, 342, 654

Rachen, J. P., \& Biermann, P. L. 1993, A\&A, 272, 161

Rieger, F. M., \& Duffy, P. 2006, ApJ, 652, 1044

Rudnick, L., Katz-Stone, D. M., \& Anderson, M. C. 1994, ApJSS, 90, 955

Rybicki, G. B., \& Lightman, A. P. 1986, Radiative Processes in Astrophysics (Wiley-VCH)

Sahayanathan, S. 2009, MNRAS, 398, L49

Sanders, R. H. 1983, ApJ, 266, 73

Schneider, P. 1993, A\&A, 278, 315

Shalchi, A., \& Dosch, A. 2009, Phys. Rev. D, 79, 3001

Sokolsky, P. et al. (HiRes Collaboration) 2009, Nucl. Phys. B Proc. Suppl., 196, 67

Stawarz, L., \& Ostrowski, M. 2002, ApJ, 578, 763

Stawarz, L., \& Petrosian, V. 2008, ApJ, 681, 1725

Stecker, F. W., Baring, M. G., \& Summerlin, E. J. 2007, ApJ, 667, L29

Summerlin, J., \& Baring, M. G. 2012, ApJ, 745, 63

Sunyaev, R. A. 1970, ApL, 7, 19

Teshima, M., Prandini, E., Bock, R., et al. 2009, Proc. 30th Int. Cosmic Ray Conf., 3, 1045

Tregillis, I. L., Jones, T. W., \& Ryu, D. 2001a, ASP Conf., 250, 336

Tregillis, I. L., Ryu, D., \& Jones, T. W. 2001b, AIP Conf., 556, 85

Tregillis, I. L., Jones, T. W., \& Ryu, D. 2004, ApJ, 601, 778

Vietri, M. 1995, ApJ, 453, 883

Walker, R. C., Ly., C., Junor, W., \& Hardee, P. J. 2008, J. Phys., 131, 2053

Wang, Y. C. 1994, Sol. Phys., 149, 347

Waxman, E. 1995, Phys. Rev. Lett., 75, 386

White, R. L. 1985, ApJ, 289, 698

Whysong, D., \& Antonucci, R. 2003, New Astron. Rev., 47, 219

Zatsepin, G. T., \& Kuzmin, V. A. 1966, J. Exp. Theoret. Phyus. Lett., 4, 78

Zavala, R. T., Taylor, G. B., \& Giovannini, G. 2007, ASP Conf., 373, 213 